\title{
Interaction of cyclodextrins with human and bovine serum albumins: A combined spectroscopic and computational investigation
}

\author{
SAPTARSHI GHOSH, BIJAN KUMAR PAUL and NITIN CHATTOPADHYAY* \\ Department of Chemistry, Jadavpur University, Kolkata 700 032, India \\ e-mail: nitin.chattopadhyay@yahoo.com
}

MS received 15 February 2014; revised 27 March 2014; accepted 28 March 2014

\begin{abstract}
Interaction of cyclodextrins (CDs) with the two most abundant proteins, namely human serum albumin (HSA) and bovine serum albumin (BSA), has been investigated using steady-state and time-resolved fluorometric techniques, circular dichroism measurements and molecular docking simulation. The study reveals that the three CDs interact differently on the fluorescence and fluorescence lifetimes of the serum albumins. However, fluorescence anisotropy and circular dichroism are not affected. Depending on their size, different CDs bind to the serum albumins in different positions, resulting in changes in the spectral behaviour of the proteins. Docking study suggests the probable binding sites of the three CDs with the proteins. Combined experimental and computational studies imply that sufficiently high concentration of CDs causes loosening of the rigid structures of these transport proteins, although their secondary structures remain intact. Thus, CDs are found to be safe for the serum proteins from the structural point of view.
\end{abstract}

Keywords. HSA; BSA; tryptophan; cyclodextrin; fluorescence; docking.

\section{Introduction}

Serum albumins, abundant in blood plasma, are among the most widely studied proteins. Structural aspects and properties of these transport proteins have been well explored. The primary structure of these transport proteins has about 580 amino acid residues and is characterized by a low content of tryptophan and a high content of cystine stabilizing a series of nine loops. The secondary structures of these serum albumins are constituted of $\sim 67 \%$ of helix of 6 turns and 17 disulfide bridges. ${ }^{1,2}$ The tertiary structure is composed of three domains, I, II, and III. Each domain is constituted of two sub-domains named A and B. ${ }^{1,2}$ Since domains II and III share a common interface, binding of a probe to domain III leads to conformational changes affecting the binding affinities to domain II and vice versa. Human and bovine serum albumins (HSA and BSA) display approximately $80 \%$ sequence homology and a repeating pattern of disulfides, which are strictly conserved. From the spectroscopic point of view, one of the main differences between the two proteins is that HSA has only one tryptophan residue (Trp-214), whereas BSA has two tryptophan residues (Trp-134 and Trp212) at different locations. ${ }^{1,2}$ From the crystallographic

\footnotetext{
*For correspondence
}

structure of HSA, it is proposed that in this protein the single tryptophan residue (Trp-214) is located in the sub-domain IIA consisting of the Lys(212)-Ala(213)$\operatorname{Trp}(214)-A l a(215)-\operatorname{Val}(216)-A l a(217)-\operatorname{Arg}(218)$ chain of the amino acid. In case of BSA, Trp-212 is located in a similar microenvironment of sub-domain IIA as the single Trp-214 in HSA, but Trp-134 is found to be localized in the second helix of the sub-domain IB, and buried in a hydrophobic pocket. ${ }^{2}$ However, this assembling of the domains can be modified, depending on the environmental conditions. Helms et al. and El-Kemary et al. have suggested that the serum proteins can adopt many conformations, ranging from a compact structure to a relaxed form. ${ }^{3,4}$ Albumins have been identified as major transport proteins in blood plasma for many compounds like drugs, fatty acids, hormones, etc. Drugs and other bioactive small molecules can bind reversibly to albumins, whereby the latter serve as carrier. Furthermore, albumins are the biomacromolecules that are involved in the maintenance of colloid-blood pressure and are implicated in facilitating transfer of many ligands across the organ-circulatory interfaces such as liver, intestine, kidney and brain. ${ }^{5}$ Fluorescenceprobe spectroscopy of proteins is one of the most powerful methodologies, yielding structural and dynamical information concerning the fluorophore environment. Since long, excited-state photophysics has been known to be exploited to explore the interaction between a 
fluorescent probe and its environment. ${ }^{6-10}$ The structures of HSA and BSA are given in scheme 1 showing different domains, sub-domains and the locations of the tryptophan units.

Cyclodextrins (CDs) are made up of glucopyranose units bound together in a ring forming a cone-shaped structure. The most common are $\alpha-, \beta$ - and $\gamma$-CDs containing 6, 7 and 8 sugar units, respectively, linked through $\alpha$-(1,4)-glycosidic bonds. Cyclodextrins can be topologically represented as toroids with a hydrophilic exterior and semi-polar interior cavity, ${ }^{11-13}$ the larger and the smaller openings of the toroid exposing secondary and primary hydroxyl groups, respectively, to the protic solvent. The interior of the toroids is not entirely hydrophobic but considerably less hydrophilic than aqueous environment, enabling them to host other hydrophobic molecules. In contrast, the exterior is sufficiently hydrophilic to impart water solubility to CDs. These two effects together lead to the formation of supramolecular host-guest inclusion complexes with a verity of hydrophobic molecules, involving noncovalent interactions in aqueous environment. ${ }^{14}$ Thus, through CD complexation, it is possible to enhance the aqueous solubility and drug bioavailability after oral administration. The structures and dimensions of the three CDs are presented in scheme 2.

CDs are often used as vehicles of many drugs for safe and targeted delivery. However, an important aspect has remained unattended. We have to ensure that addition of CDs does not affect the stability and structure of the transport proteins such as HSA and BSA in the blood stream. With this objective, in the present work, we have investigated the interaction of the serum albumins with CDs to see if there is a structural deformation of the albumin proteins upon addition of the CDs.

\section{Experimental}

\subsection{Materials}

HSA and BSA (Sigma, USA) and Tris-HCl buffer (Spectrochem, India) were used as received. As much as $1 \times 10^{-2} \mathrm{~mol} \mathrm{dm}^{-3}$ buffer solution was prepared and its $\mathrm{pH}$ was adjusted to 7.4. Stock solutions of $2 \times 10^{-3} \mathrm{~mol}$ $\mathrm{dm}^{-3}$ of both HSA and BSA proteins were prepared in this buffer. The same buffer solution was used as bulk medium throughout the study. $\alpha$-, $\beta$ - and $\gamma$-CDs (Fluka, USA) were used as received without further purification. AR-grade potassium iodide was purchased from Merck, India. Triply distilled water was used throughout the study. In all the experiments, protein concentrations were kept at $3.5 \times 10^{-6} \mathrm{~mol} \mathrm{dm}^{-3}$ and $2.5 \times$ $10^{-6} \mathrm{~mol} \mathrm{dm}^{-3}$ for HSA and BSA, respectively, except circular dichroism experiment where protein concentrations (for both HSA and BSA) were kept at a much lower value $\left(0.15 \times 10^{-6} \mathrm{~mol} \mathrm{\textrm {dm } ^ { - 3 }}\right)$ to match with the instrumental criterion.

\subsection{Methods}

Shimadzu UV-2450 absorption spectrophotometer was used for the steady-state absorption studies. Steadystate fluorescence measurements were performed with a Horiba Jobin Yvon Fluoromax-4 spectrofluorometer. Measurements of the steady-state fluorescence anisotropy were made using the same spectrofluorometer. Fluorescence anisotropy (r) is defined as

$$
r=\left(I_{V V}-G . I_{V H}\right) /\left(I_{V V}+2 G . I_{V H}\right)
$$

where $I_{V V}$ and $I_{V H}$ are the emission intensities obtained with the excitation polarizer oriented vertically and

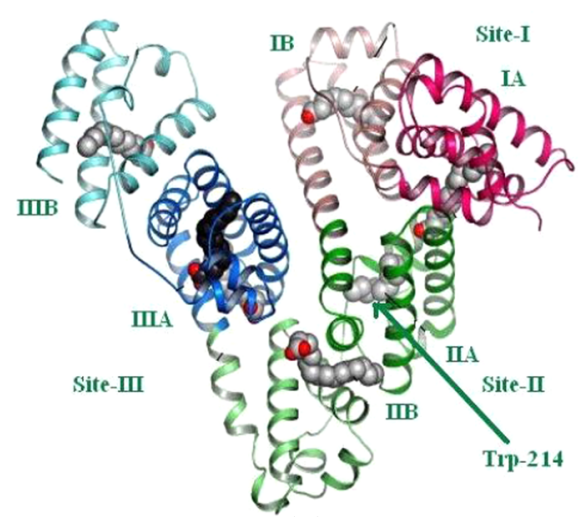

(a)

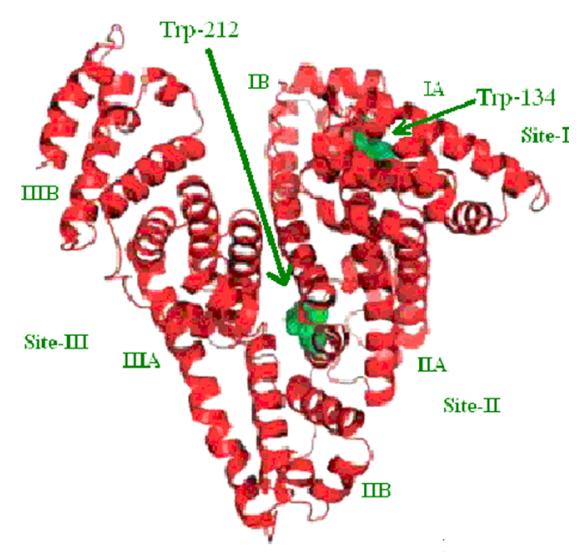

(b)

Scheme 1. Structures of (a) human serum albumin and (b) bovine serum albumin. 

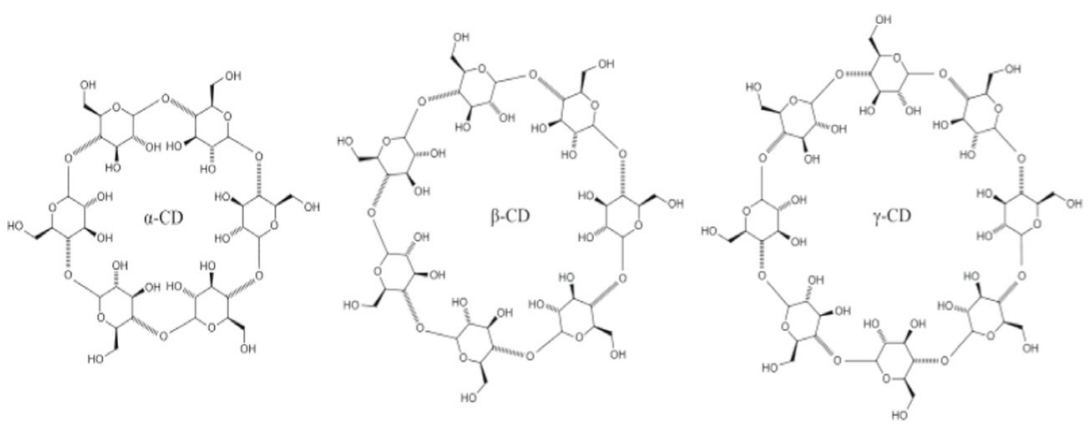

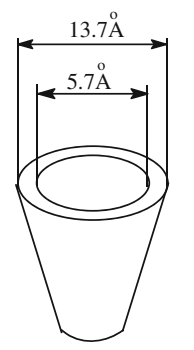

$\alpha$-cyclodextrin

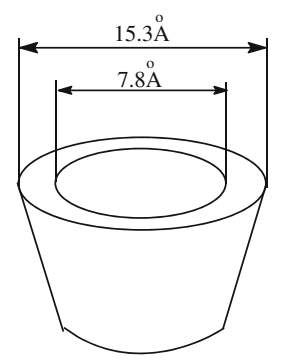

$\beta$-cyclodextrin

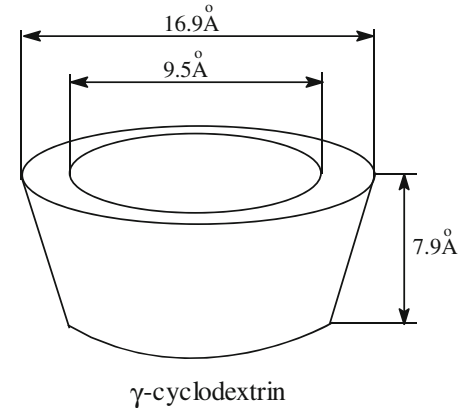

Scheme 2. Structures and dimensions of the three cyclodextrins.

emission polarizer oriented vertically and horizontally, respectively. The $\mathrm{G}$ factor is defined as ${ }^{15}$

$$
G=I_{H V} / I_{H H}
$$

where the intensities $I_{H V}$ and $I_{H H}$ refer to the vertical and horizontal positions of the emission polarizer, with the excitation polarizer being horizontal. Fluorescence lifetimes were determined from the time-resolved fluorescence decays by the method of time-correlated single photon counting (TCSPC) using excitation source at $295 \mathrm{~nm}$ (NanoLED) with TBX-04 detector (both IBH, UK). The instrument response time was $\sim 1$ ns. The instrument response function was collected by placing a scatterer (dilute micellar solution of sodium dodecyl sulfate in water) in place of the sample. The decays were analyzed using IBH DAS-6 decay analysis software. Goodness of the fits was evaluated from the $\chi^{2}$ criterion and visual inspection of the randomness of the residuals of the fitted function to the data. Mean (average) fluorescence lifetimes $\left(\tau_{\text {avg }}\right)$ for biexponential decays were calculated from the decay times $\left(\tau_{1}\right.$ and $\left.\tau_{2}\right)$ and the normalized pre-exponential factors $\left(a_{1}\right.$ and $\left.a_{2}\right)$ using the following relation:

$$
\tau_{\text {avg }}=a_{1} \tau_{1}+a_{2} \tau_{2}
$$

Circular dichroism spectra were recorded on a JASCO J-815 spectropolarimeter using a rectangular quartz cuvette of path length $1 \mathrm{~cm}$. The circular dichroism profiles were obtained employing a scan speed of $50 \mathrm{~nm} / \mathrm{min}$ and appropriate baseline corrections were made. All the experiments were performed at room temperature $(300 \mathrm{~K})$ with air-equilibrated solutions.

For molecular docking simulation, the crystal structures of the CDs were obtained from the Protein Data Bank having PDB Codes: $2 \mathrm{ZYM},{ }^{16} 2 \mathrm{Y} 4 \mathrm{~S}^{17}$ and $2 \mathrm{ZYK}^{18}$ for $\alpha-\mathrm{CD}, \beta-\mathrm{CD}$, and $\gamma-\mathrm{CD}$ respectively. The native structure of HSA was taken from the Protein Data Bank having PDB Code: 1 AO6. ${ }^{19}$ Docking simulation was performed with the AutoDock 4.2 suite of programs ${ }^{20}$ which utilizes the Lamarckian Genetic Algorithm (LGA). The grid size was set to 126, 126 and 126 along $X$-, $Y$ - and $Z$-axis with $0.414 \AA$ grid spacing. To obtain an unbiased result, blind docking simulation was performed. The AutoDocking parameters used were as follows: GA population size $=150$; maximum number of energy evaluations $=250000$; GA crossover mode $=$ two points. The PyMOL software package was used for visualization of the docked conformations. ${ }^{21}$

\section{Results and discussion}

\subsection{Steady-state absorption study}

Buffered aqueous solution of HSA gives an absorption spectrum with a band maximum at $280 \mathrm{~nm} .{ }^{22}$ Addition of CDs to the solution results in an increase in the absorbance without any appreciable shift in the absorption maximum. In all the cases, an additional little red shifted band is observed to be formed, peaking at around $325 \mathrm{~nm}$. We assign this band to the 
HSA-CD complex. The intensity of this new absorption band, however, varies with a change in the CD. Similarly, addition of CDs to the aqueous buffered solution of BSA causes increase in absorbance with no appreciable change in the position of the absorption band at $280 \mathrm{~nm} .{ }^{23}$ Here also we observe development of a new red shifted band at around $325 \mathrm{~nm}$, indicating formation of the BSA-CD complex. Like HSA, in BSA also the intensity of this new absorption band varies as the CD is changed. The absorption spectral changes of HSA and BSA with the gradual addition of $\alpha-, \beta$ - and $\gamma$-CD are shown in figure 1.

From figure 1, it is observed that with gradual addition of CDs to the serum albumin solutions, absorbance at $\sim 280 \mathrm{~nm}$, ascribed to the aromatic residues ${ }^{24}$ increases. A similar enhancement in the absorbance of the $280 \mathrm{~nm}$ band of BSA upon interaction with carbon nanotubes has recently been reported by Zhao et al. ${ }^{25} \mathrm{In}$ tune with them we ascribe the observation to a partial loosening of the serum albumin structure that eventually exposes the Trp residue to the relatively more polar aqueous buffer environment.

As is obvious from figure 1, the intensities of the new absorption bands in HSA and BSA with the addition of the three CDs do not follow any particular order. In principle, intensity of this band is supposed to depend on the concentration of the individual complex, i.e., on the binding constant $\left(K_{b}\right)$ value, as well as its molar extinction coefficient $(\varepsilon)$. The net intensity should be a reflection of the product of these two. To obtain a proper rationale to correlate the relative intensities of these absorption bands, we have determined the binding constant $\left(K_{b}\right)$ as well as the molar extinction coefficient $(\varepsilon)$ values for the complexes of serum proteins with the CDs using the Benesi-Hildebrand equation $^{26}$ given bellow.

$$
[\mathrm{SA}] / \mathrm{OD}=1 / \varepsilon K_{b}[\mathrm{CD}]+1 / \varepsilon
$$
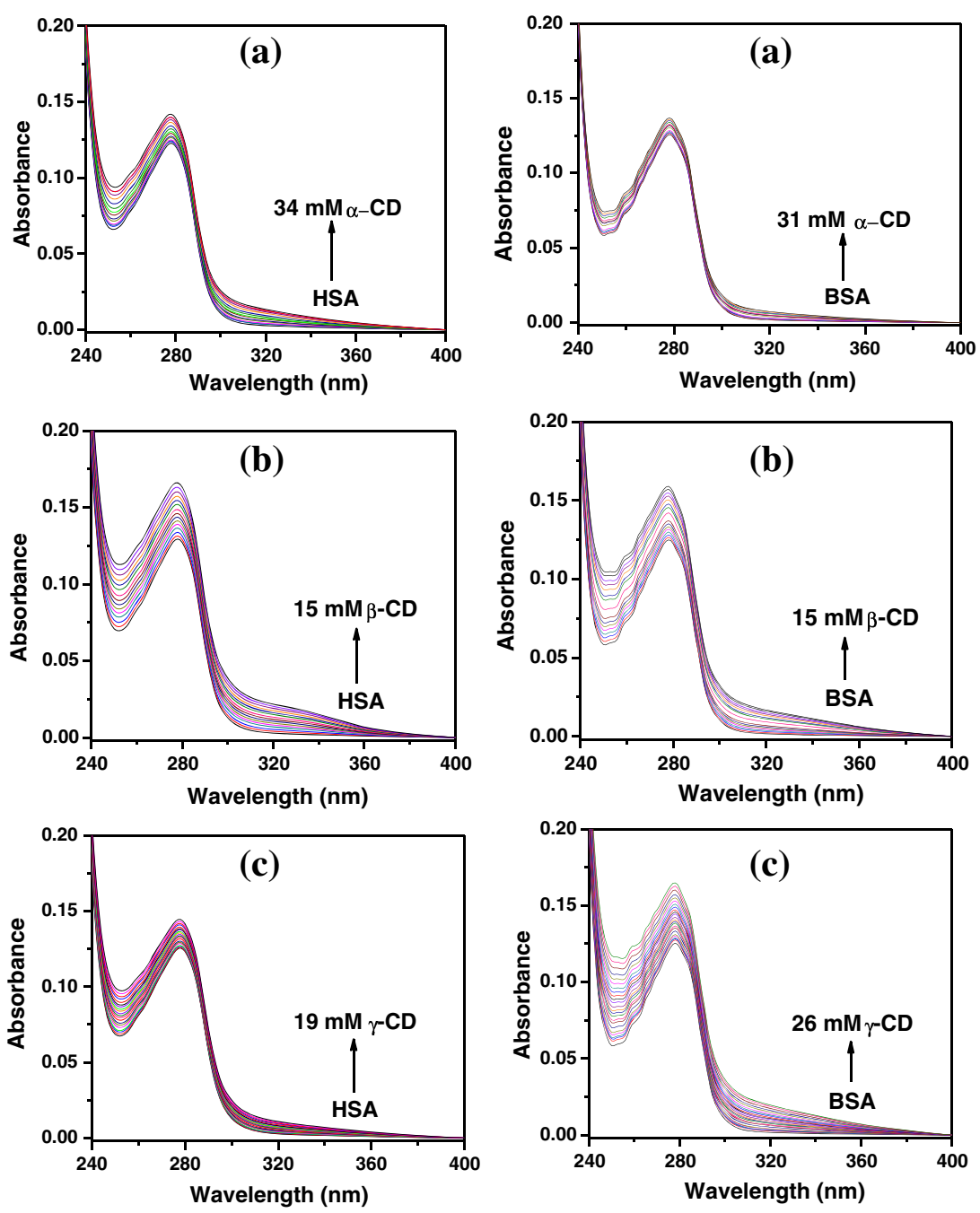

Figure 1. Absorption spectra of HSA (left panel) and BSA (right panel) as a function of (a) $\alpha-\mathrm{CD}$, (b) $\beta-\mathrm{CD}$ and (c) $\gamma-\mathrm{CD}$ concentrations. 
where $[\mathrm{SA}]$ and $[\mathrm{CD}]$ are the concentrations of the serum proteins and cyclodextrins, respectively. OD and $\varepsilon$ are the optical density and molar extinction coefficient of the protein-CD complex, respectively. From the linear plots (figures S1 and S2 given in the Supplementary information) of the [SA]/OD against $1 /[\mathrm{CD}], K_{b}$ values are determined from the ratios of the intercepts to the slopes and $\varepsilon$ values are obtained from the reciprocal of the intercepts. From the calculated $K_{b}$ values we have determined the values of the free energy changes $(\Delta \mathrm{G})$ for the $\mathrm{CD}$ complexation process by using the well-known equation (eq. 5) given below.

$$
\Delta \mathrm{G}=-\mathrm{RT} \ln K_{b}
$$

Negative $\Delta \mathrm{G}$ values imply that in all the cases the complexation processes are spontaneous. Values of $K_{b}, \Delta \mathrm{G}$ and $\varepsilon$ for all the cases are collected in table 1 .

The absorption spectral changes of the serum proteins with the gradual addition of CDs can now be well explained considering the values of $K_{b}$ and $\varepsilon$. As expected, intensities of the new absorption bands are found to follow the order of the values of the product of $K_{b}$ and $\varepsilon$ (table 1 and figure 1 ). Though the $K_{b}$ value for HSA- $\alpha$-CD complex is higher than the other two CDs, $\varepsilon$ value of this complex is reasonably low compared to the $\varepsilon$ values of HSA- $\beta$-CD and HSA- $\gamma-\mathrm{CD}$ complexes. The highest intensity of the absorption band of the HSA- $\beta$-CD complex (at $\sim 325 \mathrm{~nm}$ ) is rationalized from the highest value of the product of $K_{b}$ and $\varepsilon$ in case of complexation of HSA with $\beta$-CD. In the same way, the relative intensities of the absorption bands of the BSA-CD complexes can be rationalized from the product of the $K_{b}$ and $\varepsilon$ values of the individual complexes. It is pertinent to mention here that we have not considered the effect of aggregation of CDs since in aqueous CD solutions the fraction of molecules participating in aggregate formation is extremely low. ${ }^{27}$ For example, the mass contribution of the $\alpha$-CD-aggregates in aqueous $12 \mathrm{mM} \alpha-\mathrm{CD}$ solution does not exceed $0.8 \%$ that of $\beta$-CD in $10 \mathrm{mM} \beta$-CD solution only $0.0011 \%$ and that of $\gamma-\mathrm{CD}$ in $12 \mathrm{mM} \gamma-\mathrm{CD}$ solution only $0.02 \% .^{27}$

\subsection{Steady-state emission study}

Emission spectra of aqueous buffered solutions of HSA and BSA show single, broad and unstructured bands peaking at $\sim 345 \mathrm{~nm}$ and $\sim 350 \mathrm{~nm}$, respectively. ${ }^{28,29}$ Upon addition of the CDs, fluorescence intensities of both the proteins decrease appreciably (figure 2). In case of both HSA and BSA the extent of quenching follows the order: $\gamma-\mathrm{CD}>\beta-\mathrm{CD}>\alpha-\mathrm{CD}$. Further, we notice a detectable bathochromic shift of the emission maximum of HSA by $5 \mathrm{~nm}$ and $7 \mathrm{~nm}$ in $\beta$ - and $\gamma-\mathrm{CD}$, respectively. This shift is found to be much less for BSA ( $2 \mathrm{~nm}$ for both $\beta$ - and $\gamma-\mathrm{CD}$ ). For both the proteins, however, no detectable shift in the band positions is noticed with the addition of $\alpha$-CD. The relative extents of quenching of the fluorescence of HSA and BSA in the presence of the three CDs become more apparent when the normalized fluorescence of the protein is plotted as a function of concentration of the different CDs (figures S3 and S4 in the Supplementary information).

The remarkable quenching in the fluorescence intensities of serum albumins with the addition of CDs implies an interaction between the proteins and the CDs. It is known that fluorescence intensity of tryptophan (Trp) decreases with an increase in the solvent polarity. ${ }^{30}$ Thus, the quenching and the red shift in the emission maxima indicate that the CDs are introducing an increase in the polarity of the microenvironment around the tryptophan moiety. As will be seen from the docking study, CDs bind to different portions of the serum albumins. This seems to cause different extent of loosening of the rigid structures of the serum albumins. As a result, the tryptophan moieties get exposed to the aqueous phase to different extents leading to change in the micropolarity around tryptophan and consequent changes in the fluorometric behaviour of the serum albumins. The difference in the extent of quenching of the emission intensities of BSA compared to HSA is rationalized from the consideration of the fact that HSA contains only one tryptophan (Trp-214), whereas BSA contains two tryptophan units (Trp-212 and Trp-134) in different positions within itself. Trp-212 resides in

Table 1. Binding constant $\left(\mathrm{K}_{\mathrm{b}}\right)$, free energy change $(\Delta \mathrm{G})$ and molar extinction co-efficient $(\varepsilon)$ values for complexation of the serum proteins with cyclodextrins.

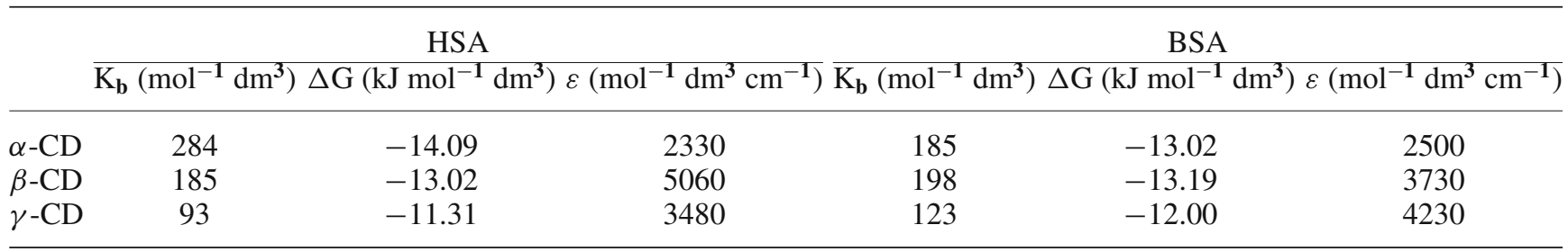



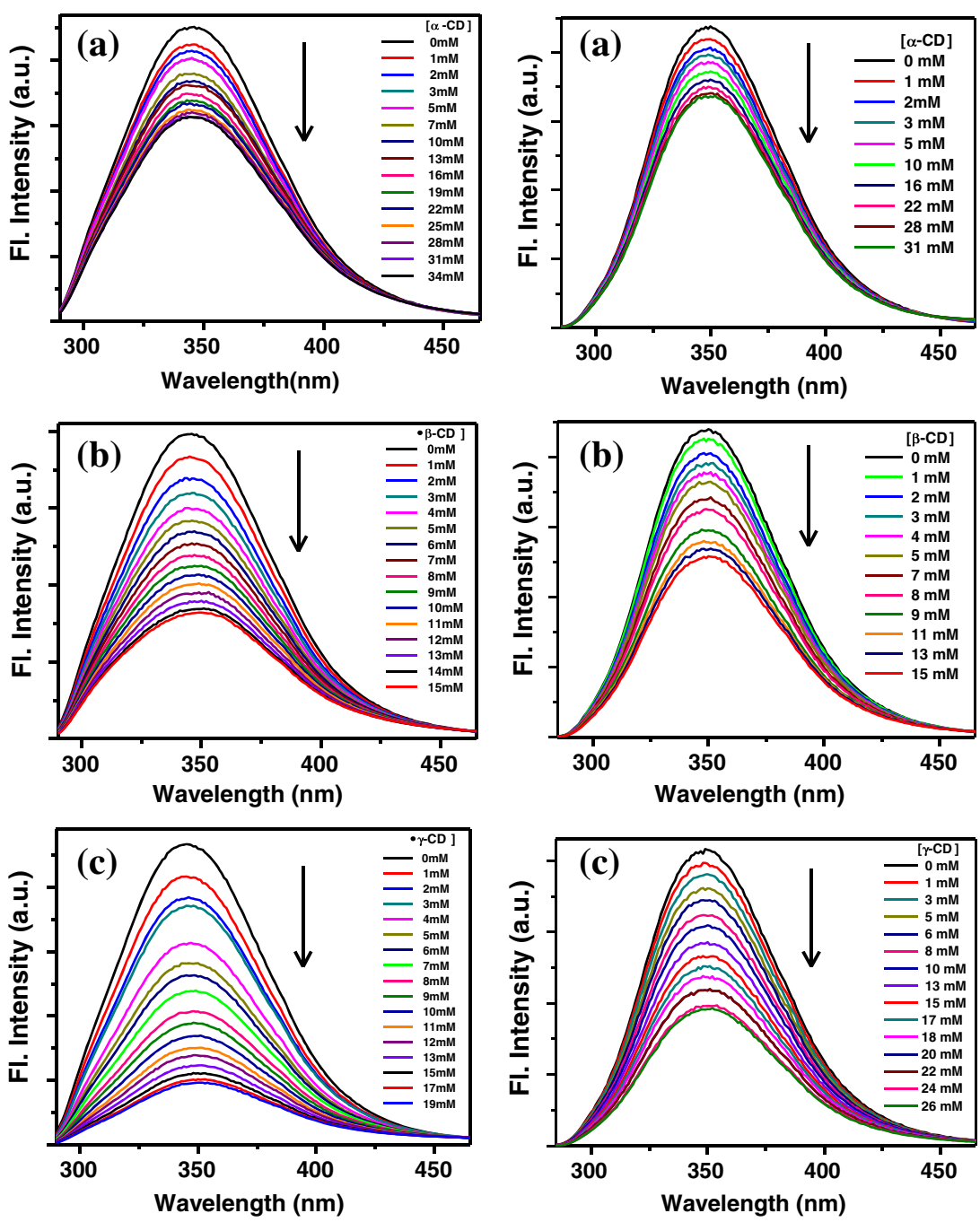

Figure 2. Variation of emission spectra of HSA (left panel) and BSA (right panel) as a function of (a) $\alpha-\mathrm{CD}$, (b) $\beta$-CD and (c) $\gamma$-CD concentration. $\lambda_{\text {exc }}=280 \mathrm{~nm}$. The concentrations of CDs are labelled in the legends.

sub-domain IIA in BSA similar to that of Trp-214 in HSA. The other tryptophan of BSA, i.e., Trp-134, is located in the sub-domain IB. Similar nature of quenching of both HSA and BSA in different CDs indicate that quenching in the emission intensities of serum albumins by $\mathrm{CD}$-complexation occurs due to the change in the micropolarity around the tryptophan moiety that resides in the similar environment (Trp-214 in HSA and Trp-212 in BSA). It appears from the experiments that Trp-134 does not report to the addition of CDs and, hence introduces in a sense, a dilution effect to the overall reduction in the fluorescence intensity of the system with the addition of the CDs. Hence, we observe less quenching of fluorescence intensity with the introduction of CDs in case of BSA.

\subsection{Time-resolved decay measurements}

Fluorescence lifetime serves as a sensitive parameter for sensing the local environment around a fluorophore, and it is sensitive to the interactions in the photo-excited state. ${ }^{31}$ It also allows one to understand the interaction between probe and proteins. ${ }^{32}$ Despite the fact that the structure of HSA is rather complicated, it has been widely studied by making use of the single Trp- 214 residue enabling the serum protein to act as an intrinsic fluorophore. In aqueous solution at neutral $\mathrm{pH}$, Trp214 exhibits multiple exponential decays, attributed to the existence of rotational conformational isomers, called rotamers. ${ }^{15}$ In the present work, we have studied the effect of addition of CDs to a buffered solution of HSA by measuring the lifetimes of Trp-214 under 
various concentrations of CDs. Depending on the available excitation source, for lifetime measurements the samples are excited at $295 \mathrm{~nm}$ and the fluorescence decays of HSA in the absence and presence of different CDs are monitored at the respective emission maxima. Figure 3 shows representative decays of HSA in different environments. When aqueous buffered solution of HSA is photo-excited at $295 \mathrm{~nm}$, the decay profile shows a biexponential pattern yielding lifetime values of $4.5 \mathrm{~ns}\left(\tau_{1}\right)$ and $8.25 \mathrm{~ns}\left(\tau_{2}\right) .{ }^{32}$ With gradual addition of the CDs the lifetime values are reduced, in conformity with the quenching of the steady-state fluorescence intensity. Consistent with the steady-state fluorometric experiment, the decrease in the lifetime values of HSA in the three CDs are found to follow the order $\gamma$ $\mathrm{CD}>\beta-\mathrm{CD}>\alpha-\mathrm{CD}$. Similarly to the steady-state fluorescence, the fluorescence lifetime of tryptophan is also known to decrease with an increase in the polarity of the medium. ${ }^{33}$ It appears that complexation of HSA with the CDs results in the access of some water molecules in the periphery of the fluorophore Trp-214 unit.

Aqueous buffered solution of BSA also shows a biexponential decay with lifetime values $4.5 \mathrm{~ns}\left(\tau_{1}\right)$ and $7.5 \mathrm{~ns}\left(\tau_{2}\right) .{ }^{34}$ With gradual addition of the CDs to the buffered solution of BSA, the values of both shorter and longer lifetimes get reduced following the same order as we observe for HSA, i.e., $\gamma-\mathrm{CD}>\beta-\mathrm{CD}>\alpha$-CD. Consistent with the steady-state emission study, here also we observe lesser extent of decrease in the lifetime values than in case of HSA. This can be rationalized by the dilution effect coming from the extra tryptophan, i.e., Trp-134 of BSA, which is hardly affected by the action of the CDs, as discussed earlier.

The analyzed fluorescence lifetime data of both the albumins in the presence of varying concentrations of $\alpha-, \beta$ - and $\gamma$-CDs are collected in tables S1-S6 (given in the Supplementary information). The normalized lifetime values ( $\tau_{1}$ and $\tau_{2}$ ) of HSA in presence of varying concentrations of the CDs are plotted (figure 4) for better understanding. A similar figure (figure S5) for BSA is provided in the Supplementary information.

Time-resolved studies, thus, corroborate the results of the steady-state studies and hence strengthen the proposition that introduction of the CDs to the serum albumins causes formation of CD-protein complex, resulting in loosening of the protein structures that allows some of the water molecules to come to the near vicinity of the responsible tryptophan moiety. So, the microenvironment around the tryptophan becomes more polar than it was before the introduction of the
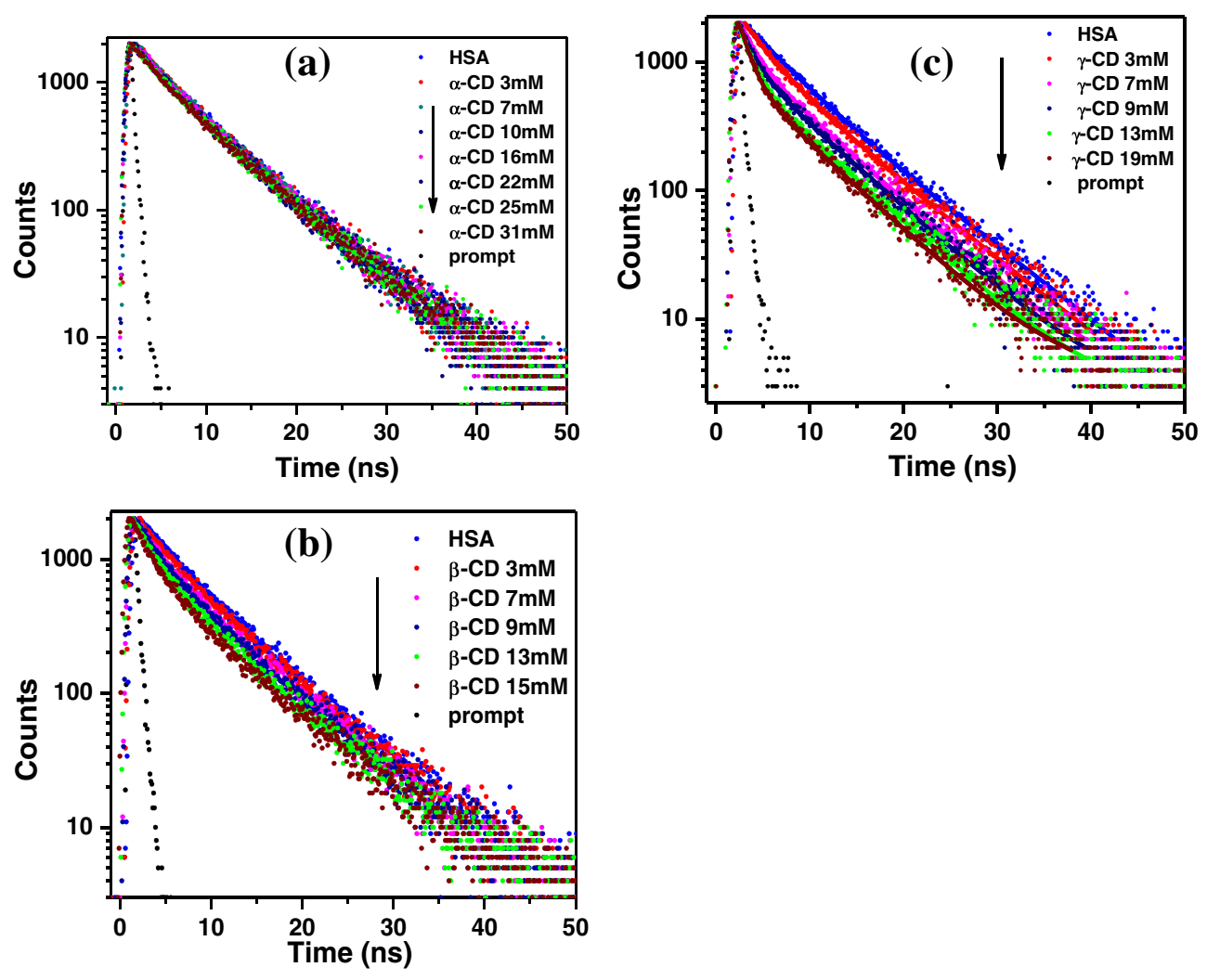

Figure 3. Time-resolved fluorescence decays of HSA in (a) $\alpha$-CD, (b) $\beta$-CD and (c) $\gamma$-CD $\left(\lambda_{\text {exc }}=295 \mathrm{~nm}\right)$. The sharp profiles on the left are the lamp profiles. Fitted lines to the decay data are omitted in (A) and (B) to avoid clumsiness of the figures. 

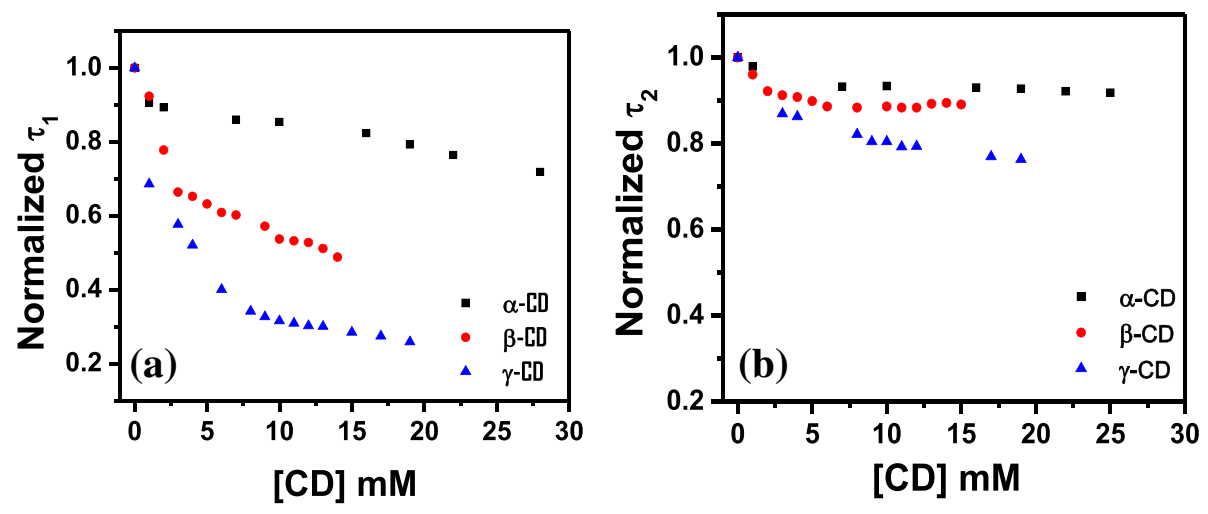

Figure 4. Variation of normalized shorter lifetime (a) and longer lifetime (b) components of HSA in three CDs. $\lambda_{\mathrm{exc}}=295 \mathrm{~nm}$.

CDs. As we see from the steady-state and time-resolved studies for both the proteins, effect of three CDs is not same, which is expected from the difference in the dimensions of the CDs. Depending on the dimensions of the openings of the CDs, they bind to different portions of the serum albumins, exerting differential effects. This provokes us to find out the probable location of binding of the three CDs from molecular docking study to be described in a forthcoming section.

\subsection{Steady-state fluorescence quenching study}

Steady-state quenching experiment is an important tool in fluorescence spectroscopy to understand the degree of exposure of the fluorophore towards aqueous phase..$^{35,36}$ To substantiate whether or not the CDs are forcing the tryptophan moiety in the serum albumins to be exposed more to the aqueous phase, we have performed steady-state quenching studies using $\mathrm{KI}$ as a quencher. We have done the experiment in the absence and in the presence of $\beta$-CD only to look at the changes in the value of the Stern-Volmer constant $\left(\mathrm{K}_{\mathrm{SV}}\right)$ that indicates the degree of exposure of the fluorophore to the quencher. Larger the value of $\mathrm{K}_{\mathrm{SV}}$, higher the degree of exposure. Stern-Volmer constants $\left(\mathrm{K}_{\mathrm{SV}} \mathrm{s}\right)$ can be determined from the slopes of the plots of $F_{0} / F$ against concentration of the quencher. ${ }^{15}$

$$
F_{0} / F=1+\mathrm{K}_{\mathrm{SV}}[\mathrm{Q}]
$$

where $F_{0}$ and $F$ are the fluorescence intensities in the absence and in the presence of the quencher, respectively, and $[\mathrm{Q}]$ is the quencher concentration. Interestingly, for neither of the two serum albumins we get a linear Stern-Volmer plot (figure 5). This non-linearity of the Stern-Volmer plot is ascribed to the biphasic quenching of tryptophan by the quencher. ${ }^{37-40}$ The first phase of quenching is ascribed to quenching by the iodide ions that originally resides near the tryptophan (quasi-static quenching), whereas the second phase is due to quenching by free diffusing iodide ions (dynamic quenching). In tune with Möller and Denicola, ${ }^{37}$ we have used the modified Stern-Volmer equation to tackle with the biphasic quenching.

$$
F_{0} /\left(F_{0}-F\right)=\left(F_{0} / F_{0}^{\prime}\right)\left(1 / \mathrm{K}_{\mathrm{SV}}[\mathrm{Q}]\right)+\left(F_{0} / F_{0}^{\prime}\right)
$$

From the linear plot of $F_{0} /\left(F_{0}-F\right)$ against 1/[Q] (figure 6), $\mathrm{K}_{\mathrm{SV}}$ values are obtained from the ratios of the intercepts to the slopes and are tabulated in table 2.

Similar observations in the quenching study for both the serum albumins suggest that the tryptophans, which are in similar environment in the two serum albumins (Trp-214 of HSA and Trp-212 of BSA), are involved in the quenching process. The second tryptophan in BSA, i.e., Trp-134, remains unaffected by the quencher, which is consistent with the observation by Möller and Denicola. ${ }^{37}$

The quenching constant values of the serum albumins in the presence of $\beta$-CD are slightly higher than those in its absence. The higher $\mathrm{K}_{\mathrm{SV}}$ values in the presence of $\mathrm{CD}$ indicate that with the introduction of $\beta-\mathrm{CD}$, the tryptophan moieties (Trp-214 of HSA and Trp-212 of BSA) of the serum albumins get exposed to the aqueous phase, although only partially.

\subsection{Steady-state fluorescence anisotropy study}

With an objective to check whether the interaction of the serum proteins with the CDs leads to a reduction in the restriction on the rotational motion of the tryptophan or not we have performed the steady-state fluorescence anisotropy study ${ }^{41}$ Fluorescence anisotropy reflects the extent of restriction imposed by the microenvironment 

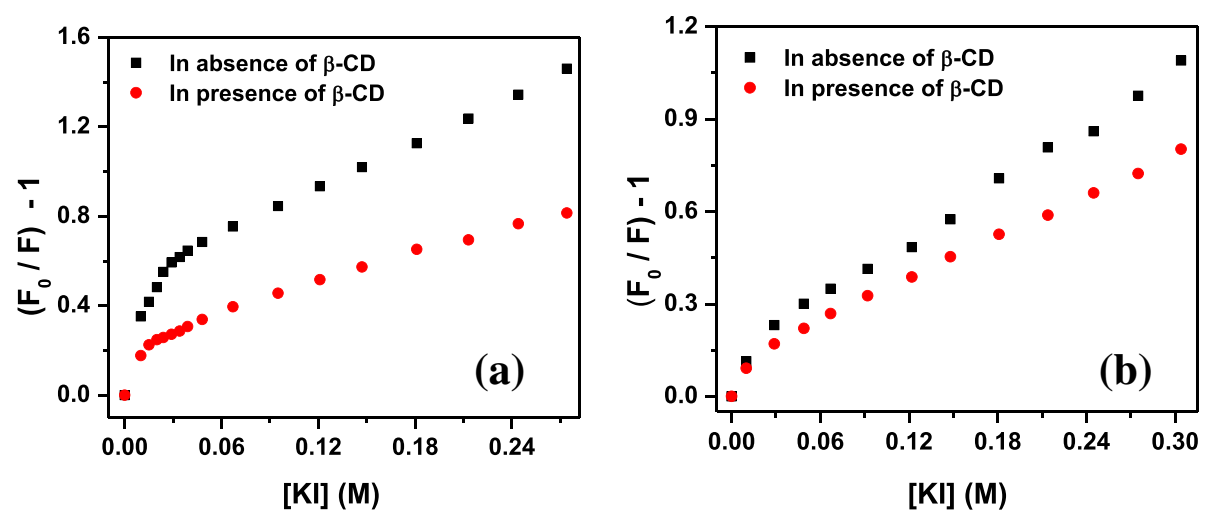

Figure 5. Stern-Volmer plots for the fluorescence quenching of tryptophan in (A) HSA and (B) BSA in the absence and presence of $10 \mathrm{mM} \beta$-CD.

on the dynamic properties of the probe, and hence can be exploited in assessing the motional information of the probe in various microheterogeneous environments. An increase in the fluorescence anisotropy signifies an increase in the motional restriction on the probe and therefore indicates an increase in the rigidity of the surrounding environment. We have monitored the steady-state fluorescence anisotropy of tryptophan residues of HSA and BSA as a function of concentration of different CDs to check whether the CDs encapsulate the tryptophan units of the serum albumins within the hydrophobic cavities or not. If CDs encapsulate the tryptophan unit within the cavities, we would expect an increase in the anisotropy value since in that case tryptophan unit will face a more constrained environment. But we observe inappreciable change in the anisotropy values of both the serum albumins in the presence of all the CDs (figure 7).

It is pertinent to mention here that even in the absence of the CDs the fluorescence anisotropies of both HSA

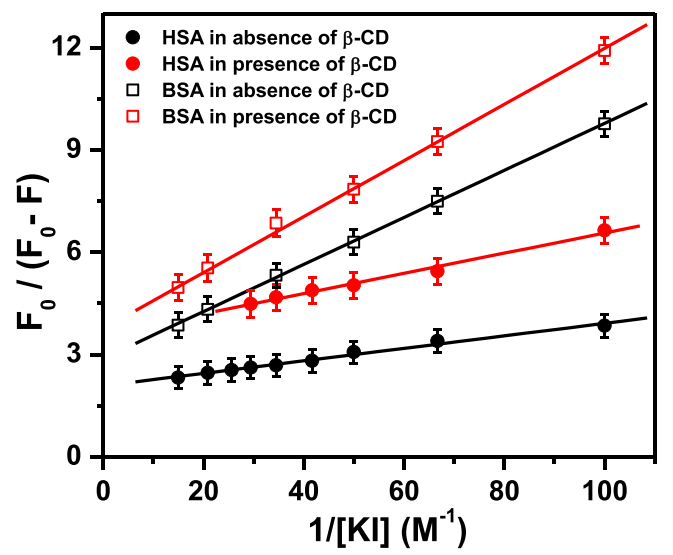

Figure 6. Modified Stern-Volmer plot for quenching of fluorescence of HSA and BSA in absence and presence of $10 \mathrm{mM} \beta$-CD. and BSA are found to be rather high. The higher fluorescence anisotropy values ( 0.10 and 0.085 for HSA and BSA, respectively) of tryptophan in the proteins can be attributed to the compact structure of the proteins encompassing the tryptophan/s imparting restriction on its/their rotational motion. The steady-state anisotropy measurements, however, reveal that the CDs neither impose any motional restriction on the tryptophan moiety of the serum albumins nor induce any reduction in the restriction, already present in the proteins. This implies that neither are the tryptophan units incorporated into the cavities of the CDs nor are the structure of the proteins loosened to an extent to impart more rotational freedom to the tryptophan moieties in the presence of the CDs. However, absorption and steadystate and time-resolved fluorometric studies definitely establish an interaction between the CDs and the serum albumins. Thus, it seems that CDs bind to the serum albumins at positions away from the tryptophan units, which appears reasonable since serum albumins, being macromolecules, do possess multiple binding sites. The possible binding sites of the two serum proteins are predicted from the docking study, to be discussed later.

\subsection{Circular dichroism study}

Circular dichroism spectroscopy is widely used to monitor the structure, conformation and stability of the

Table 2. $\mathrm{K}_{\mathrm{Sv}}$ values in different media

$\begin{array}{cc}\begin{array}{c}\mathrm{K}_{\mathbf{S V}}\left(\mathrm{mol}^{-\mathbf{1}} \mathrm{dm}^{\mathbf{3}}\right) \\ \text { in absence of } \\ 10 \mathrm{mM} \beta \text {-CD }\end{array} & \begin{array}{c}\mathrm{K}_{\mathbf{S V}}\left(\mathrm{mol}^{-\mathbf{1}} \mathrm{dm}^{\mathbf{3}}\right) \\ \text { in presence of } \\ 10 \mathrm{mM} \beta \text {-CD }\end{array} \\ 113.66 & 121.49 \\ 41.78 & 45.71\end{array}$



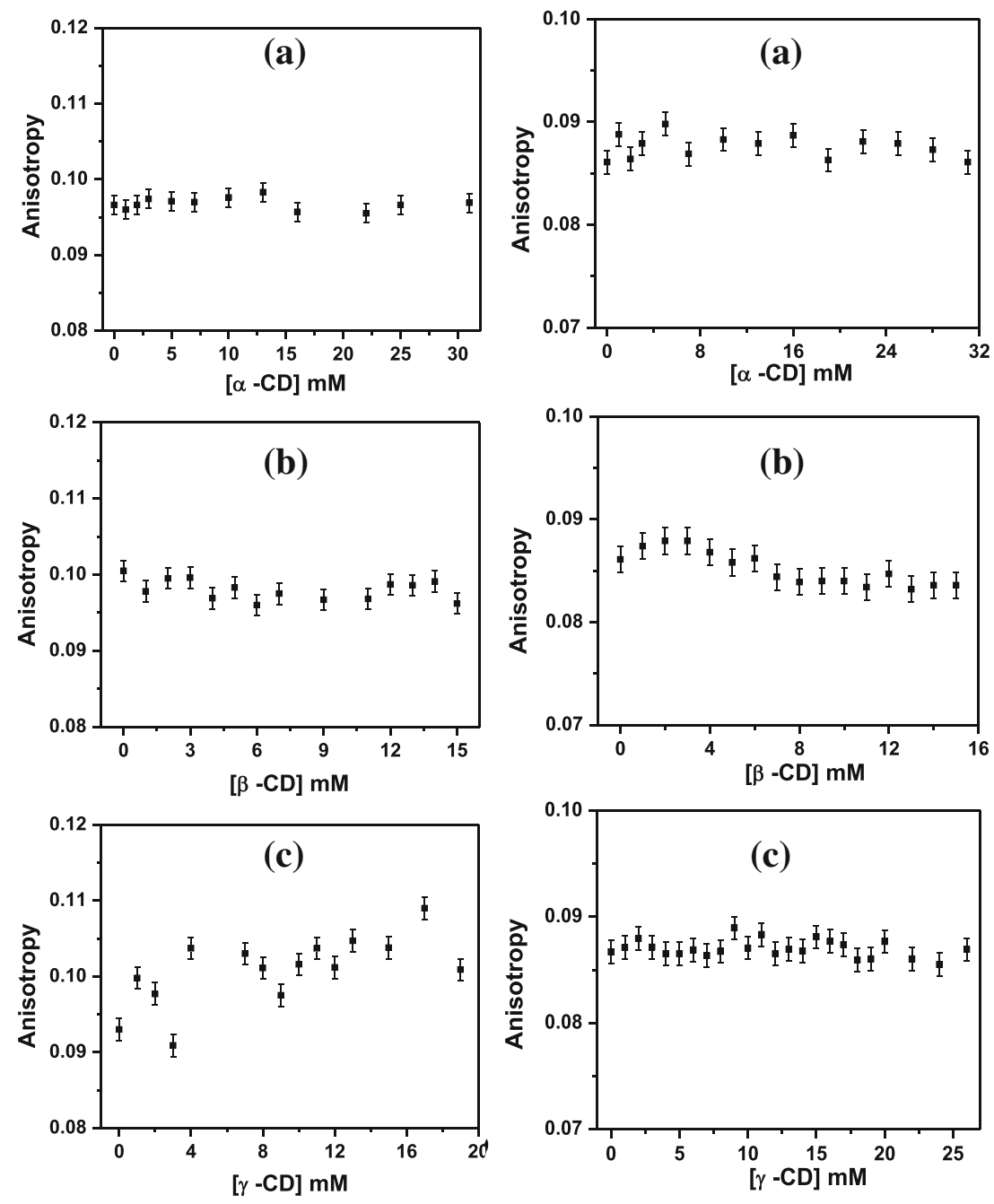

Figure 7. Variation of fluorescence anisotropy of HSA (left panel) and BSA (right panel) as a function of concentration of (a) $\alpha-\mathrm{CD}$, (b) $\beta$-CD and (c) $\gamma$-CD.

proteins. ${ }^{42-44}$ It probes the secondary structure of proteins although it does not provide the details regarding the precise structure. Fluorescence techniques are rather more sensitive in deciphering small changes at molecular level. ${ }^{28}$ To decipher the structural and conformational changes, if any, brought in by the added cyclodextrins, we have performed the circular dichroism spectral studies of the serum albumins in the presence of varying concentrations of the three cyclodextrins. It is evident from figure 8 that the circular dichroism spectra of HSA and BSA show two minima at 208 and $222 \mathrm{~nm}$, which is a signature of the presence of $\alpha$-helix in the proteins under study. ${ }^{42-44}$ As already stated, due to the instrumental restriction we had to take lesser concentrations of the serum proteins for the circular dichroism study, as compared to the concentrations used for the other studies. Keeping parity with the relative concentrations of the albumins to $\mathrm{CDs}$, here we have added lesser amounts of CDs. Addition of different CDs is found to cause imperceptible change in the circular dichroism spectrum of the serum proteins, which implies that CDs are not affecting the secondary structure of the serum albumins to a detectable level. It is clear from the circular dichroism spectra that CDs are not altering the protein structures as much to be probed by circular dichroism spectroscopy. This implies that CDs cannot penetrate inside the serum albumins. Rather, they bind to the surface of the proteins. Steady-state fluorescence anisotropy study also reveals that tryptophans in the proteins do not get encapsulated inside the nanocavities of the CDs, consistent with the results obtained from the circular dichroism spectroscopy. This confirms that CDs are unable to change the conformation of the serum proteins to an extent to leave a signature on their circular dichroism spectra. This seems rational from the 

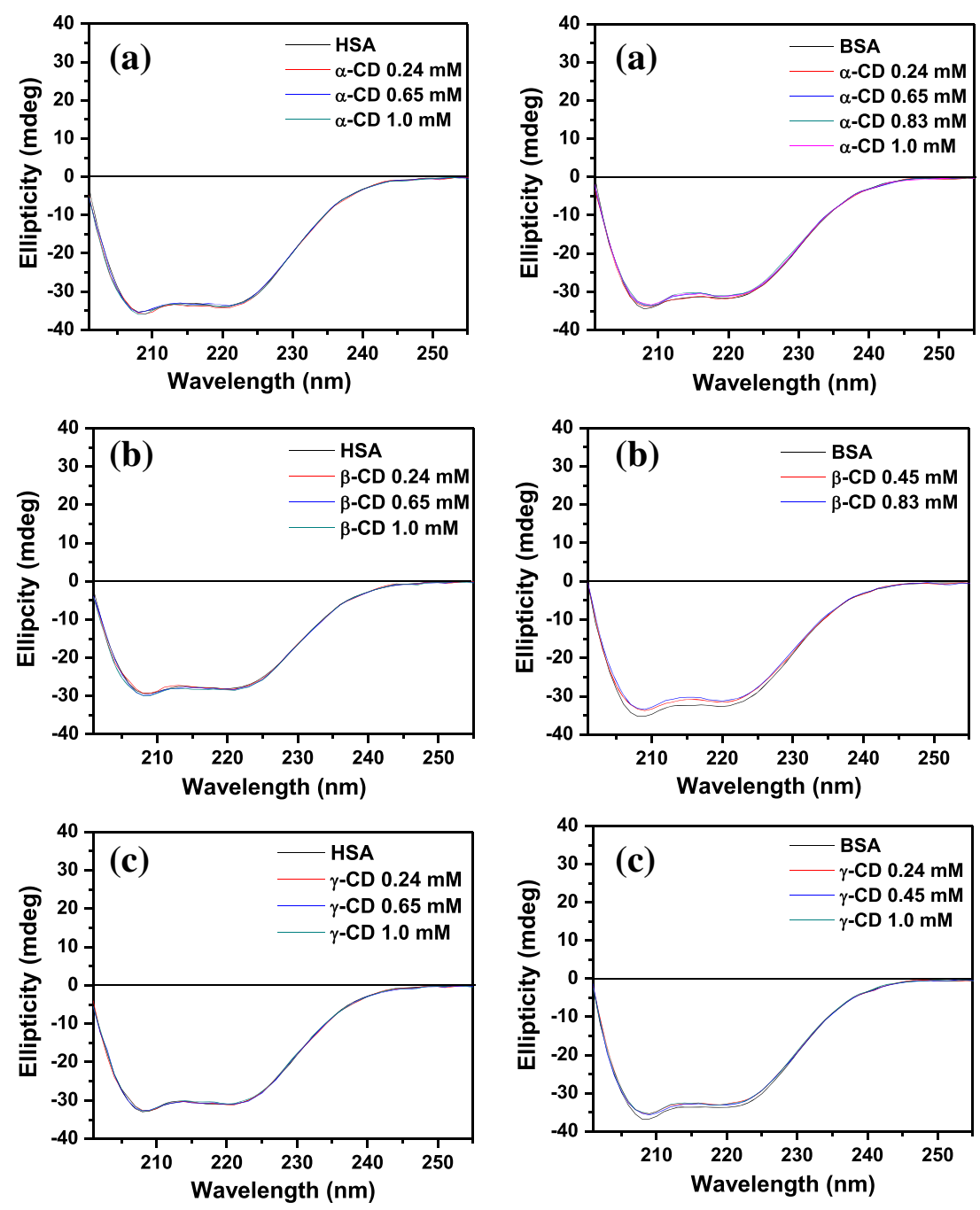

Figure 8. Circular dichroism spectra of HSA (left panel) and BSA (right panel) as a function of concentration of (a) $\alpha-\mathrm{CD}$, (b) $\beta$-CD and (c) $\gamma$-CD. The concentrations of CDs used are labeled in the legends.

standpoint of the structures of the serum albumins composed of a single polypeptide chain. The proteins contain 17 disulfide bridges, which are located at regular intervals in the protein molecules. Thus, in addition to the general compact folded nature of the native protein, the flexibility of serum albumin molecules is considerably restricted by these 17 disulfide bridges ${ }^{45}$ that is also evident from the higher anisotropy values of tryptophan in the proteins. Hence, when CDs interact with the serum proteins in a freely diffusing medium, the compact native structures of the proteins are not expected to be unfolded to a considerable extent. Rather, CDs should interact, preferably, along the surface of the proteins. Blind molecular docking simulations have been exploited to decipher the possible locations of binding.

\subsection{Molecular modeling: Blind docking simulation}

To understand the binding action of CDs to the two albumin proteins, knowledge of proper binding location of the CDs onto the proteins is essential. With this objective, we have performed docking simulation study with HSA only according to the protocol described in section 2.2. As HSA and BSA display approximately $80 \%$ sequence homology ${ }^{34}$ it is expected that CDs bind to the serum albumins in similar sites. Thus, molecular docking simulation with only HSA should be enough to understand the binding locations of the serum proteins. To obtain an unbiased result in this respect, the AutoDock-based blind docking simulation has been employed. The strategy of AutoDock-based blind docking includes a search over the entire surface of the 


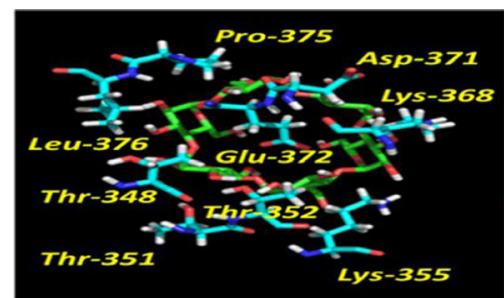

Protein residues in near vicinity (within 4.0 $A$ ) of $\alpha-C D$

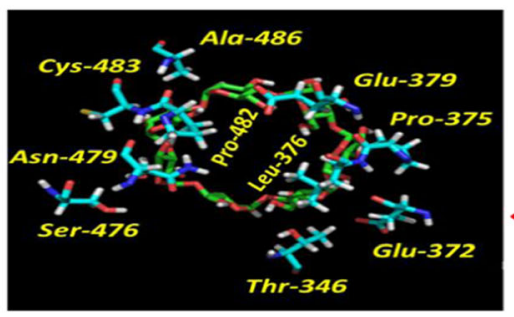

Protein residues in near vicinity (within 4.0 Ä) of $\beta-C D$

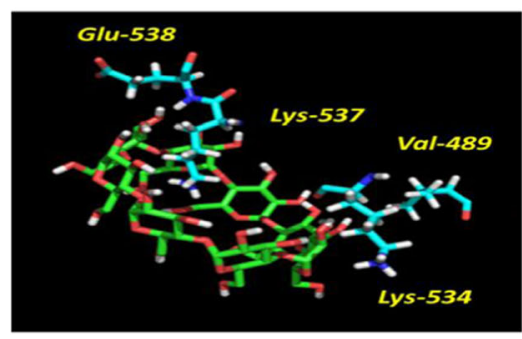

Protein residues in near vicinity (within 4.0 $A$ ) of $v$-CD

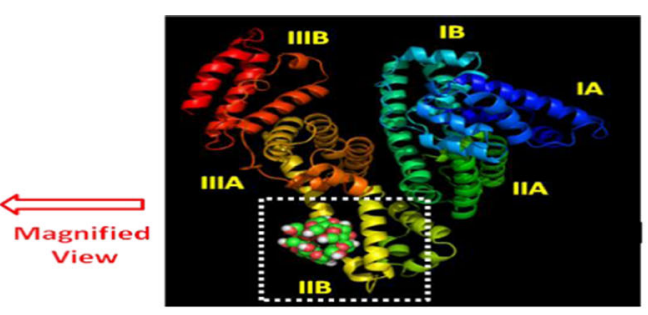

(a)

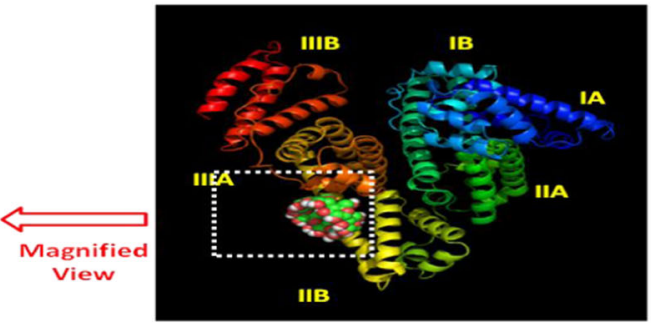

(b)

HSA: $\beta-C D$ Docked conformation

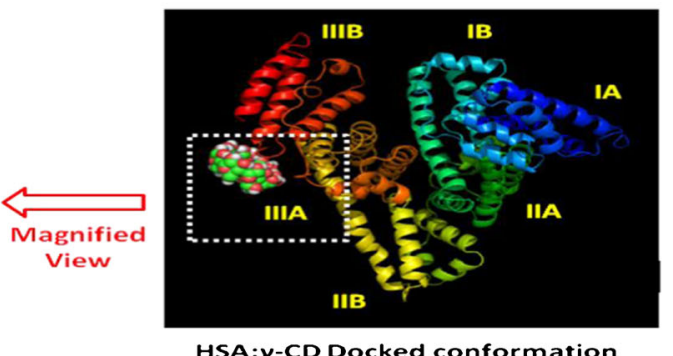

(c)

Figure 9. Stereo view of docked conformation of (A) $\alpha-C D$, (B) $\beta$-CD and (C) $\gamma$ $\mathrm{CD}$ with the protein HSA. Left panel in each case displays the protein residues in near vicinity (within $4.0 \AA$ ) of the CDs over a molecular surface presentation of the protein. The pictures have been prepared by PyMOL software package.

protein for binding sites and simultaneously optimizing the conformations of the peptides. ${ }^{20,46-48}$ This leads to an unbiased result, and hence has fairly been described as "very encouraging" in a recent review. ${ }^{48}$ The docked results are presented in figure 9. Docking study with human serum albumin (HSA) implies that the three different CDs bind to three different portions of HSA, considerably away from the tryptophan moiety. Docking study suggests that both $\alpha$ - and $\beta$-CD bind in the subdomain IIB, whereas $\gamma$-CD binds in the sub-domain IIIA. This binding of CDs in the sub-domain IIB or IIIA affects the spatial conformation of the tryptophan unit located in the sub-domain IIA as domains II and III share a common interface. The binding of CDs with the serum albumins depends on the cavity sizes of the CDs. The hydrophobic interaction plays a major role in the protein-CD complexation process. Recently, Aachmann et al. have reported a similar type of hydrophobic interaction between protein and CD. ${ }^{49}$
They have also suggested that CDs bind to solventaccessible aromatic side chains on the surface of proteins. The hydrophobic residues get encapsulated in the cavities of the CDs, depending on the diameter of the opening of the $\mathrm{CD}$ cavities, resulting in a change in the energy landscape of the protein-CD systems, taking it to another conformational state. The described experiments suggest that the CD complexation causes little loosening of the compact protein structure and this might be the factor responsible for exposing the tryptophan moiety more towards the aqueous phase.

\section{Conclusions}

The present work establishes the interactions of three well-known cyclodextrins with two major transport proteins, HSA and BSA, through vivid experimental studies as well as molecular docking simulation. Depending 
on their cavity sizes, CDs bind to these transport proteins in different positions. This binding leads to a change in the polarity around the micro-environment of the intrinsic fluorophore of these proteins, i.e., the tryptophan moiety. Absorption, steady-state and timeresolved fluorometric and fluorescence quenching studies reveal that at high concentration of CDs the protein structure is loosened considerably, which, apprehensively, might affect the normal function of these proteins. Fluorescence anisotropy and circular dichroism studies, however, reveal insignificant structural change induced by the CDs. Circular dichroism study confirms no detectable change in the secondary structure of the proteins with the introduction of the CDs. Thus, although CDs can be considered as physiologically safe so far as their action on the serum proteins is concerned, their use at higher concentrations might degrade the transport ability of the serum proteins towards carrying drugs.

\section{Supplementary Information}

Benesi-Hildebrand plots for both HSA and BSA, plot of normalized fluorescence of the proteins against the concentration of the different CDs, the analyzed fluorescence lifetime data of both the albumins in the presence of varying concentrations of the three CDs and variation of normalized lifetime values of BSA in the presence of varying concentrations three CDs are presented in the Supplementary information (see www.ias. ac.in/chemsci).

\section{Acknowledgements}

Financial supports from the Department of Science and Technology (DST) and Department of Biotechnology (DBT), Government of India, are gratefully acknowledged. S G thanks University Grants Commission (UGC) for his fellowship.

\section{References}

1. Min H X and Carter D C 1992 Nature 358209

2. Peters T 1985 Serum albumin, advances in protein chemistry (New York: Academic Press)

3. Helms M K, Peterson C E, Bhagavan N V and Jameson D M 1997 FEBS Lett. 40867

4. El-Kemary M, Gil M and Douhal A 2007 J. Med. Chem. $\mathbf{5 0} 2896$

5. Hu Y J, Liu Y and Xiao X-H 2009 Biomacromolecules 10517

6. Barreleiro P C A and Lindman B 2003 J. Phys. Chem. B 1076208
7. DemchenSko A P 1992 In Topics in fluorescence spectroscopy: Biochemical applications J R Lakowicz (ed.) (New York: Plenum)

8. Cardenas M, Schillen K, Pebalk D, Nylander T and Lindman B 2005 Biomacromolecules 6832

9. Haldar B, Chakrabarty A, Mallick A, Mandal M C, Das P and Chattopadhyay N 2006 Langmuir 223514

10. Mallick A, Bera S C, Maiti S, Haldar B and Chattopadhyay N 2004 Biophys. Chem. 1129

11. Connors K A 1997 Chem. Rev. 971325

12. Szejtli J 1998 Chem. Rev. 981743

13. Liu L and Guo Q-X 2002 J. Inclusion Phenom. Macrocycl. Chem. $\mathbf{4 2} 1$

14. Rekharsky M V and Inoue Y 1998 Chem. Rev. 98 1875

15. Lakowicz J R 2006 Principles of fluorescence spectroscopy (ed.) (New York: Springer)

16. Matsumoto N, Yamada M, Kurakata Y, Yoshida H, Kamitori S, Nishikawa A and Tonozuka T 2009 Febs. J. 2763008

17. Vester-Christensen M B, AbouHachem M, Syensson B and Henriksen A 2010 J. Mol. Biol. 403739

18. Tonozuka T, Sogawa A, Yamada M, Matsumoto N, Yoshida H, Kamitori S, Ichikawa K, Mizuno M, Nishikawa A and Sakano Y 2007 Febs J. 2742109

19. Sugio S, Kashima A, Mochizuki S, Noda M and Kobayashi K 1999 Proetin Engg. 12439

20. Morris G M, Goodsell D S, Halliday R S, Huey R, Hart W E, Belew R K and Olson A J 1998 J. Comput. Chem. 191639

21. De Lano W L 2002 The PyMOL molecular graphics system (San Carlos, CA, USA: De Lano Scientific)

22. Keshavarz M 2009 J. Phys. Theor. Chem. 6113

23. Togashi D M, Ryder A G, Mc Mahon D, Dunne P and McManus J 2007 Proc. SPIE-OSA Biomed. Opt. 66281

24. Liu R, Sun F, Zhang L, Zong W, Zhao X, Wang L, Wu R and Hao X 2009 Sci. Total Environ. 4074184

25. Zhao X, Liu R, Chi Z, Teng Y and Qin P 2010 J. Phys. Chem. B 1145625

26. Benesi H A and Hildebrand J H 1949 J. Am. Chem. Soc. 712703

27. Messner M, Kurkov S V, Jansook P and Loftsson T 2010 Intl. J. Pharmaceutics 387199

28. Anand U, Jash C and Mukherjee S 2010 J. Phys. Chem. B 11415839

29. Maity A, Mukherjee P, Das T, Ghosh P and Purkayastha P 2012 Spectrochimica Acta Part A 92382

30. Muiño P L and Callis P R 2009 J. Phys. Chem. B 113 2572

31. Das P, Mallick A, Haldar B, Chakrabarty A and Chattopadhyay N 2006 J. Chem. Phys. 1250445161

32. Patel S and Datta A 2007 J. Phys. Chem. B 11110557

33. Vekshin N, Vincent M and Gallay J 1992 Chem. Phys. Lett. 199459

34. Gelamo E L, Silva C H T P, Imasato H and Tabak M 2002 Biochim. Biophys. Acta 159484

35. Bose D, Ghosh D, Das P, Girigoswami A, Sarkar D and Chattopadhyay N 2010 Chem. Phys. Lipids $\mathbf{1 6 3}$ 94

36. Jana B, Ghosh S and Chattopadhyay N 2013 J. Photochem. Photobiol. B: Biol. 1261 
37. Möller M and Denicola A 2002 Biochem. Mol. Biol. Educ. 30175

38. Lehrer S S and Leavis P C 1978 Methods Enzymol 49 222

39. Noel J K and Hunter M J 1972 J. Biol. Chem. 2477391

40. Lehrer S S 1978 Biochemistry 103254

41. Chakrabarty A, Mallick A, Haldar B, Das P and Chattopadhyay N 2007 Biomacromolecules 8920

42. Moriyama Y and Takeda K 1999 Langmuir 152003

43. Moriyama $Y$ and Takeda K 2005 Langmuir 215524

44. Parker W and Song P S 1992 Biophys. J. 611435
45. Brown J R 1977 In Albumin structure, function, and uses V M Rosenoer, M Oratz, M A Rothschild (ed.) (Oxford: Pergamon Press)

46. Hetenyi C and Van Der Spoel D 2006 FEBS Lett. 580 1447

47. Paul B K, Ray D and Guchhait N 2012 Phys. Chem. Chem. Phys. 148892

48. Campbell S J, Gold N D, Jackson R M and Westhead D R 2003 Curr Opin. Struct. Biol. 13389

49. Aachmann F L, Otzen D E, Larsen K L and Wimmer R 2003 Protein Eng. 16905 